\title{
Computational Analysis of the Influence of Dissolution-Diffusion and Specific Binding on Drug Transport in the Arterial Wall following Stent-based Delivery
}

\author{
Javier Escuer ${ }^{1}$, Estefanía Peña ${ }^{1,2}$, Sean McGinty ${ }^{3}$, Miguel Ángel Martínez ${ }^{1,2}$ \\ ${ }^{1}$ Applied Mechanics and Bioengineering (AMB) \\ Aragón Institute of Engineering Research (I3A) \\ UniversityZaragoza, Mariano Esquillor s/n, 50018, Zaragoza, Spain. \\ Tel.+34-976762707, e-mail: jescuer@unizar.es \\ ${ }^{2}$ Biomedical Research Networking Center in Bioengineering, Biomaterials and Nanomedicine (CIBER-BBN) \\ ${ }^{3}$ Division of Biomedical Engineering, University of Glasgow, UK.
}

\begin{abstract}
A computational model of the transport of drugs eluted from a drug-eluting stent (DES) in the arterial wall is developed to study the influence of considering a non-linear model of dissolutiondiffusion in the polymeric strut coating of the stent and a non-linear saturable reversible reaction model in the tissue to describe drug specific and nonspecific binding.
\end{abstract}

\section{Introduction}

Cardiovascular diseases (CVDs) are the number one cause of mortality in developed countries. Atherosclerosis, the major precursor of CVD, is characterized by the accumulation of lipids and fibrous elements inside the arteries causing a progressive reduction in the lumen available for blood flow (stenosis). Stents have become the most successful devices to treat advanced atherosclerotic lesions in arteries. However, one of the main issues with these interventions is the development of restenosis, the recurrence of stenosis, defined as a progressive luminal renarrowing of the blood vessel that was previously opened up via PCI, either with or without stent implantation. The coating of these devices with antiproliferative substances to reduce this effect is now standard, although such drugs can also delay re-endothelialization of the intima. The drug release strategy is therefore a key determinant of drug-eluting stent (DES) efficacy.

\section{Materials and Methods}

We develop a 2D axisymmetric computational model of coupled stent drug release and redistribution in the arterial wall, similar to that employed by Bozsak et al. [1] but with two important differences: we consider a non-linear model of dissolution-diffusion in the polymeric strut coating of the stent [2] and a non-linear saturable reversible reaction model in the tissue to describe drug specific binding to receptors and drug nonspecific binding to general extracellular matrix sites [3]. The computational geometry consists of an idealized straight segment of a healthy coronary artery with ten circular struts half-embedded in the tissue, located $0.7 \mathrm{~mm}$ center to center apart. In the lumen, blood is treated as a Newtonian fluid and blood flow is assumed to be steady, laminar and incompressible flow and is modelled by timeindependent Navier-Stokes equations. The arterial wall is modelled as a multilayer anisotropic porous structure distinguishing two different domains: intima or subendothelial space (SES) and media. Darcy's law is used to calculate plasma filtration through the tissue and convection-diffusion-reaction equations are used to model drug transport through blood, intima and media. Endothelium and internal elastic lamina are considered as semipermeable membranes and the flux across them is described by Kedem-Katchalsky equations. Model input parameters were derived from experimental data available in the literature. The commercial software package COMSOL Multiphysics 5.0 (COMSOL $\mathrm{AB}$, Burlington, MA, USA) was used to create the computational geometry and to solve numerically the partial differential equations by means of the finite element method (FEM).

\section{Results}

As example of the obtained results the temporal evolution of the normalized mean concentration (NMC) for tow different drugs, sirolimus and paclitaxel, is presented (Figure 1).

\section{Conclusions}

A numerical model which takes into account the combined effects of diffusion, dissolution and 
solubility in the polymer coating and two different types of binding in the tissue is presented in this work. This more realistic model is able to help design and develop new drug delivery systems (DDS) much more efficiently.

\section{REFERENCES}

[1]. BOZSAK, F., CHOMAZ, J.M., BARAKAT, A.I. Modeling the transport of drugs eluted from stents: physical phenomena driving drug distribution in the arterial wall. Biomechanics and Modelling in Mechanobiology. 2014, 13(2), 327-347.
[2]. McGINTY, S., PONTRELLI, G. A general model of coupled drug release and tissue absortion dor drug delivery devices. Journal of Controlled Release. 2015, 217:327-336

[3]. TZAFRIRI, A.R, GROOTHUIS, A., PRICE, G.S., EDELMAN, E.R. Stent elution rate determines drug deposition and receptor-mediated effects. Journal of Controlled Release. 2012, 161(3):918-926.

\section{FIGURES}

\section{SIROLIMUS}

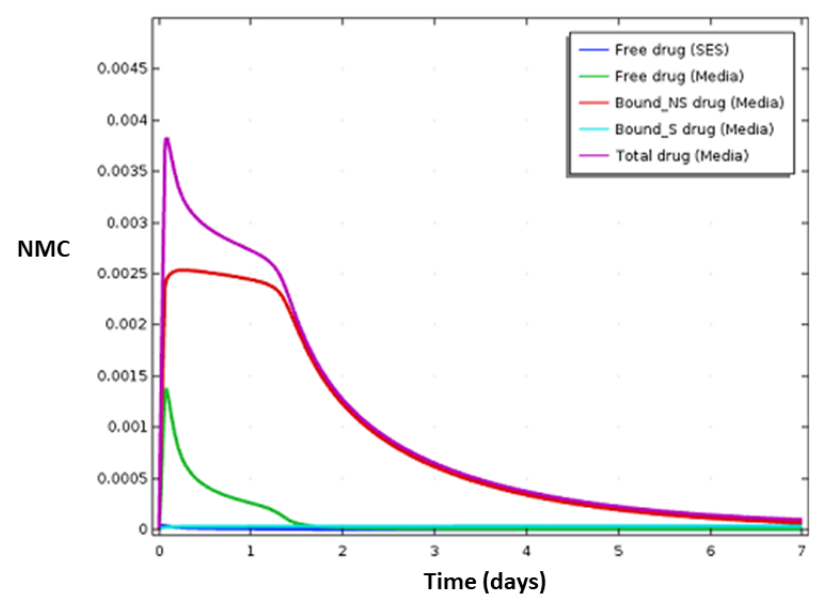

PACLITAXEL

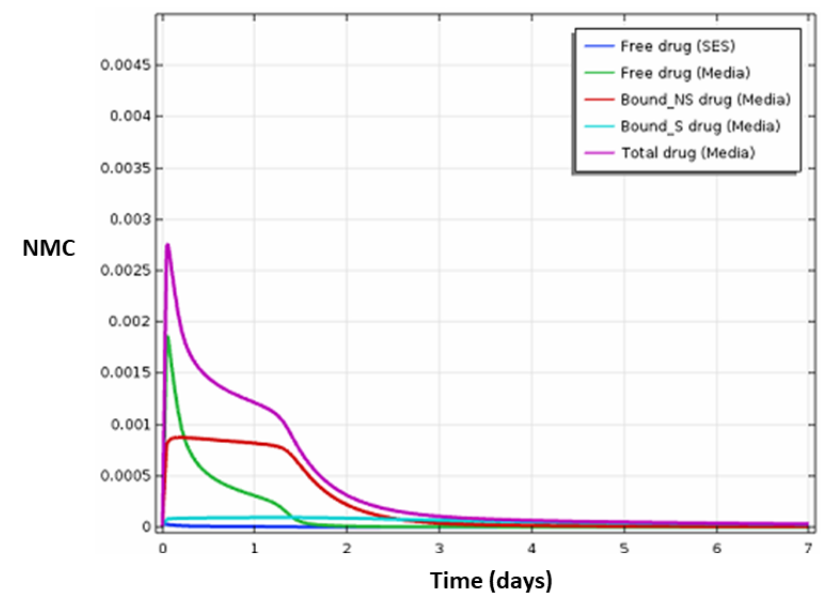

Figure 1. Temporal evolution of the normalized mean concentration (NMC) in the SES and the media for two different drugs: sirolimus and paclitaxel. 